\title{
BOUNDED PROJECTIONS, DUALITY, AND MULTIPLIERS IN SPACES OF ANALYTIC FUNCTIONS $\left(^{1}\right)$
}

\author{
BY \\ A. L. SHIELDS AND D. L. WILLIAMS
}

\begin{abstract}
Let $\varphi$ and $\psi$ be positive continuous functions on $[0,1)$ with $\varphi(r) \rightarrow 0$ as $r \rightarrow 1$ and $\int_{0}^{1} \psi(r) d r<\infty$. Denote by $A_{0}(\varphi)$ and $A_{\infty}(\varphi)$ the Banach spaces of functions $f$ analytic in the open unit disc $D$ with $|f(z)| \varphi(|z|)=o(1)$ and $|f(z)| \varphi(|z|)=O(1)$, $|z| \rightarrow 1$, respectively. In both spaces $\|f\|_{\varphi}=\sup _{D}|f(z)| \varphi(|z|)$. Let $\boldsymbol{A}^{1}(\psi)$ denote the space of functions analytic in $D$ with $\|f\|_{\psi}=\iint_{D}|f(z)| \psi(|z|) d x d y<\infty$. The spaces $A_{0}(\varphi), A^{1}(\psi)$, and $A_{\infty}(\varphi)$ are identified in the obvious way with closed subspaces of $C_{0}(D), L^{1}(D)$, and $L^{\infty}(D)$, respectively. For a large class of weight functions $\varphi, \psi$ which go to zero at least as fast as some power of $(1-r)$ but no faster than some other power of $(1-r)$, we exhibit bounded projections from $C_{0}(D)$ onto $A_{0}(\varphi)$, from $L^{1}(D)$ onto $A^{1}(\psi)$, and from $L^{\infty}(D)$ onto $A_{\infty}(\varphi)$. Using these projections, we show that the dual of $A_{0}(\varphi)$ is topologically isomorphic to $A^{1}(\psi)$ for an appropriate, but not unique choice of $\psi$. In addition, $A_{\infty}(\varphi)$ is topologically isomorphic to the dual of $A^{1}(\psi)$. As an application of the above, the coefficient multipliers of $A_{0}(\varphi), A^{1}(\psi)$, and $A_{\infty}(\varphi)$ are characterized. Finally, we give an example of a weight function pair $\varphi, \psi$ for which some of the above results fail.
\end{abstract}

We study certain Banach spaces of analytic functions in the unit disc $D$. For example, let $A_{\infty}$ denote the space of functions satisfying $|f(z)|=O\left((1-|z|)^{-1}\right)$, let $A_{0}$ denote the subspace with $O$ replaced by $o$, and let $A^{1}$ denote the space of functions with $\iint|f| d x d y<\infty$. We may regard $A_{0}$ as a subspace of $C_{0}(D)$, the continuous functions on the disc that vanish on the boundary. We first show that there are bounded projections from $C_{0}(D)$ onto $A_{0}$, from $L^{1}(D)$ onto $A^{1}$, and from $L^{\infty}(D)$ onto $A_{\infty}$ (Theorem 1). Using this, Lindenstrauss and Pełczyński have shown that $A^{1}$ is topologically isomorphic to $l^{1}$.

Using this we show in Theorem 2 that $A^{1}$ may be identified with the conjugate space of $A_{0}$, and $A_{\infty}$ with the conjugate space of $A^{1}$. (For the special case indicated above this result is in Duren, Romberg, and Shields [2, Theorems 7 and 11], but our proof applies to more general weight functions, as indicated below.)

Finally, we find all sequences $\left\{\lambda_{n}\right\}$ such that $\sum \lambda_{n} a_{n} z^{n} \in A_{\infty}$ whenever $\sum a_{n} z^{n} \in A_{\infty}$, and likewise for $A_{0}$ and $A^{1}$ (Theorem 3).

Presented to the Society, January 22, 1970 under the title Spaces of analytic functions with weighted supremum norms; received by the editors July 22, 1970 and, in revised form, March 1, 1971.

AMS 1970 subject classifications. Primary 46E15; Secondary 30A98.

Key words and phrases. Banach spaces, analytic functions, projections, topological direct sums, multiplier transforms.

(1) This work was supported in part by the National Science Foundation.

Copyright (C) 1972, American Mathematical Society 
Our proofs work when $1-r$ is replaced by a weight function $\varphi(r)$ that goes to zero at least as fast as some power of $(1-r)$, but no faster than some other power of $(1-r)$ (see (6) for a precise statement), and when $A^{1}$ is suitably modified (see (7)). It seems likely that the results are not true for all weight functions $\varphi$, however (see §4).

1. Preliminaries. Let $D$ denote the open unit disc in the complex plane; let $A(D)$ denote the set of all analytic functions in $D$. Let $\varphi(r), \psi(r)(0 \leqq r<1)$ be positive and continuous, with

$$
\lim _{r \rightarrow 1} \varphi(r)=0 \text { and } \int_{0}^{1} \psi(r) d r<\infty
$$

We extend $\varphi$ and $\psi$ to $D$ by defining $\varphi(z)=\varphi(|z|)$ and $\psi(z)=\psi(|z|)$. For $f \in A(D)$ we define

$$
\begin{gathered}
\|f\|_{\varphi}=\sup _{z \in D}|f(z)| \varphi(z)=\sup _{0 \leqq r<1} M_{\infty}(f, r) \varphi(r), \\
\|f\|_{\psi}=\int|f| \psi d \sigma=2 \int_{0}^{1} M_{1}(f, r) \psi(r) r d r
\end{gathered}
$$

where

$$
M_{1}(f, r)=\frac{1}{2 \pi} \int_{0}^{2 \pi}\left|f\left(r e^{i \theta}\right)\right| d \theta, \quad M_{\infty}(f, r)=\max _{|z|=r}|f(z)|
$$

and $\sigma$ denotes normalized Lebesgue measure on $D$. We shall be concerned with the following spaces of analytic functions.

$$
\begin{aligned}
& A_{\infty}=A_{\infty}(\varphi)=\left\{f \in A(D):\|f\|_{\varphi}<\infty\right\}, \\
& A_{0}=A_{0}(\varphi)=\left\{f \in A(D): \lim _{r \rightarrow 1} M_{\infty}(f, r) \varphi(r)=0\right\}, \\
& A^{1}=A^{1}(\psi)=\left\{f \in A(D):\|f\|_{\psi}<\infty\right\} .
\end{aligned}
$$

Clearly $A_{0}(\varphi) \subset A_{\infty}(\varphi)$ so we may use the norm $\|f\|_{\varphi}$ on $A_{0}(\varphi)$. These three spaces are all normed linear spaces with the indicated norms.

LEMMA 1. Let $A$ denote any of the above three normed spaces.

(i) If $B$ is a bounded subset of $A$, then the functions in $B$ are uniformly bounded on each compact subset of $D$.

(ii) If $\left\{f_{n}\right\}$ is a Cauchy sequence in $A$, then it converges uniformly on each compact subset of $D$.

(iii) Point evaluation at any point of $D$ is a bounded linear functional on $A$.

(iv) $A$ is a Banach space.

(v) $A_{0}$ is a closed subspace of $A_{\infty}$. 
Proof. (i) This is obvious for $A_{0}$ and $A_{\infty}$. In $A^{1}$ we have, if $|z| \leqq R<1$,

$$
f(z)=\frac{1}{2 \pi i} \int_{|\zeta|=\rho} f(\zeta)(\zeta-z)^{-1} d \zeta
$$

where $\rho=\frac{1}{2}(1+R)$. Hence

$$
|f(z)| \leqq \frac{1}{2 \pi} \int_{0}^{2 \pi}\left|f\left(\rho e^{i t}\right)\right|(\rho-R)^{-1} d t=2(1-R)^{-1} M_{1}(f, \rho) .
$$

Also,

$$
M_{1}(f, \rho) \int_{\rho}^{1} \psi(r) d r \leqq \int_{\rho}^{1} M_{1}(f, r) \psi(r) d r<\|f\|_{\psi}
$$

since $M_{1}(f, r)$ is an increasing function of $r$. The result follows.

(ii) and (iii) follow from the above estimate for $f(z)$.

(iv) It is only necessary to establish completeness, and this is easy for $A_{\infty}$ and $A_{0}$. If $\left\{f_{n}\right\}$ is a Cauchy sequence in $A^{1}$, then it converges uniformly on compact sets to an analytic function $f$, by (ii). Also, $f \in A^{1}$ by Fatou's lemma. Thus $A^{1}$ is complete.

(v) This follows from (iv).

Let $C_{0}(D)$ denote the Banach space of continuous functions on the closed disc that vanish on the boundary, with the supremum norm. Also, let $L^{1}(D)$ and $L^{\infty}(D)$ denote, respectively, the usual Banach spaces of integrable and essentially bounded measurable functions associated with Lebesgue measure on $D$. The maps

$$
T_{0}: A_{0}(\varphi) \rightarrow C_{0}(D), \quad T_{\infty}: A_{\infty}(\varphi) \rightarrow L^{\infty}(D), \quad T_{1}: A^{1}(\psi) \rightarrow L^{1}(D),
$$

defined by $T_{0} f=\varphi f, T_{\infty} f=\varphi f, T_{1} g=\psi g$ are isometries. We use the following notation for the ranges of these operators.

Notation. $T A_{0}=T_{0} A_{0}(\varphi), T A^{1}=T_{1} A^{1}(\psi), T A_{\infty}=T_{\infty} A_{\infty}(\varphi)$.

Thus $T A_{0}$ is a subspace of $C_{0}(D), T A^{1}$ is a subspace of $L^{1}(D)$, and $T A_{\infty}$ is a subspace of $L^{\infty}(D)$. These subspaces are closed by Lemma 1 .

Let $M(D)$ denote the Banach space of complex-valued, bounded Borel measures on $D$ with the variation norm. The map

$$
M: A^{1}(\psi) \rightarrow M(D)
$$

defined by $M g=g \psi d \sigma$ is an isometry of $A^{1}$ onto a closed subspace of $M(D)$, which we denote by $M A^{1}$.

$L^{\infty}(D)$ and $M(D)$ are conjugate Banach spaces and hence have $w^{*}$ topologies. These topologies induce topologies on $A_{\infty}(\varphi)$ and $A^{1}(\psi)$ which we shall call the $w^{*}$ topologies on these spaces.

LEMMA 2. $T A_{\infty}$ is a $w^{*}$ closed subspace of $L^{\infty}(D)$.

For the proof see Lemma 1 of [7].

Define $f_{r}(z)=f(r z), 0 \leqq r<1$, for $f \in A(D)$. 
Lemma 3. (i) For $f \in A^{1}$ or $A_{0}, f_{\rho} \rightarrow f$ in norm as $\rho \rightarrow 1$.

(ii) For $f \in A_{\infty}, f_{\rho} \rightarrow f\left(w^{*}\right)$.

(iii) The polynomials are dense in $A^{1}$ and in $A_{0}$.

(iv) The polynomials are $w^{*}$ dense in $A_{\infty}$; in fact, each $f \in A_{\infty}$ is the $w^{*}$ limit of a sequence of polynomials.

Proof. (i) This is obvious for $A_{0}$. For $f \in A^{1}$ and $\varepsilon>0$ choose $R<1$ so that

$$
\frac{1}{2} \int_{|z|>R}|f(z)| \psi(z) d \sigma=\int_{R}^{1} M_{1}(f, r) \psi(r) r d r<\varepsilon .
$$

Since $M_{1}\left(f_{\rho}, r\right)=M_{1}(f, \rho r) \leqq M_{1}(f, r)$ we have

$$
\frac{1}{2} \int_{|z|>R}\left|f_{\rho}(z)\right| \psi(z) d \sigma<\varepsilon \quad(0<\rho<1) .
$$

Choose $\rho$ so that $\left|f-f_{\rho}\right|<\varepsilon$ on $|z| \leqq R$. Then

$$
\frac{1}{2} \int\left|f-f_{\rho}\right| \psi d \sigma<\frac{\varepsilon}{2} \int_{|z| \leqq R} \psi d \sigma+2 \varepsilon<\varepsilon\left(\|\psi\|_{1}+2\right) .
$$

(ii) We must show that

$$
\lim _{\rho \rightarrow 1} \int \varphi f_{\rho} h d \sigma=\int \varphi f h d \sigma
$$

for all $h \in L^{1}(D)$, and this follows from the dominated convergence theorem.

(iii) In either $A_{0}$ or $A^{1}$, if $\varepsilon>0$ is given, choose $\rho$ so that $\left\|f-f_{\rho}\right\|<\varepsilon$; this is possible by (i). Now choose a polynomial $p$ so that $\left|f_{\rho}-p\right|<\varepsilon$ on all of $D$. The result follows.

(iv) Let $f \in A_{\infty}$ and let $\sigma_{n}$ denote the $n$th Cesàro mean of the partial sums of the Taylor series of $f$. Then $M_{\infty}\left(\sigma_{n}, r\right) \leqq M_{\infty}(f, r)$ (see [5, Erstes Kapitel, §1, Satz 1]). Hence $\left\|\sigma_{n}\right\|_{\varphi} \leqq\|f\|_{\varphi}$, and so the sequence of $\varphi \sigma_{n}$ converges pointwise and boundedly to $\varphi f$. From the dominated convergence theorem we have

$$
\lim \int \varphi \sigma_{n} h d \sigma=\int \varphi f h d \sigma
$$

for all $h \in L^{1}(D)$, which was to be proved.

LeMmA 4. For $\alpha>-1$ and $n=0,1, \ldots$,

$$
\int_{0}^{1} r^{n}(1-r)^{\alpha} d r=n ! /(\alpha+1)(\alpha+2) \cdots(\alpha+n+1) .
$$

Proof. The proof is by induction on $n$. The result is trivial for $n=0$. Assume that it holds for $n=k$ and all $\alpha>-1$. Integrating by parts,

$$
\int_{0}^{1} r^{k+1}(1-r)^{\alpha} d r=(k+1)(\alpha+1)^{-1} \int_{0}^{1} r^{k}(1-r)^{\alpha+1} d r
$$

Now apply the induction hypothesis to the right side. 
LeMma 5. For $\alpha>0$ and $0<r<1$,

$$
\int_{0}^{2 \pi}\left|1-r e^{i t}\right|^{-1-\alpha} d t=O\left((1-r)^{-\alpha}\right) .
$$

For a proof see Duren [1, Chapter $4, \S 6]$.

LEMMA 6. For $\gamma>-1$ and $m>1+\gamma$ we have

$$
\int_{0}^{1}(1-\rho r)^{-m}(1-r)^{\gamma} d r \leqq c(1-\rho)^{1+\gamma-m}, \quad 0<\rho<1 .
$$

Proof. Integrating by parts,

$$
\begin{aligned}
\int_{0}^{1}(1-\rho r)^{-m}(1-r)^{\gamma} d r & =\frac{1}{\gamma+1}+\frac{m}{\gamma+1} \rho \int_{0}^{1}(1-\rho r)^{-m-1}(1-r)^{\gamma+1} d r \\
& \leqq c_{1}+c_{2} \rho \int_{0}^{1}(1-\rho r)^{-m+\gamma} d r
\end{aligned}
$$

since $(1-r)^{\gamma+1} \leqq(1-\rho r)^{\gamma+1}$. The result follows.

We now specialize the weight functions $\varphi$ and $\psi$.

DeFINITION. The function $\varphi$ will be called normal if there exist $k>\varepsilon>0$ and $r_{0}<1$, such that

$$
\frac{\varphi(r)}{(1-r)^{\varepsilon}} \searrow 0 \text { and } \frac{\varphi(r)}{(1-r)^{k}} \nearrow \infty \quad\left(r_{0} \leqq r, r \rightarrow 1^{-}\right) \text {. }
$$

Note that $k$ and $\varepsilon$ are not uniquely determined by $\varphi$.

DEFINITION. The functions $\{\varphi, \psi\}$ will be called a normal pair if $\varphi$ is normal and if, for some $k$ satisfying (6), there exists $\alpha>k-1$ such that

$$
\varphi(r) \psi(r)=\left(1-r^{2}\right)^{\alpha}, \quad 0 \leqq r<1 .
$$

Lemma 7. If $\varphi$ is normal then there exists $\psi$ such that $\{\varphi, \psi\}$ is a normal pair.

Proof. Choose $k$ such that $\varepsilon(r)=(1-r)^{k} / \varphi(r) \rightarrow 0$. Then

$$
\psi(r)=\left(1-r^{2}\right)^{\alpha} / \varphi(r)=\varepsilon(r)\left(1-r^{2}\right)^{\alpha} /(1-r)^{k}
$$

will be integrable if we choose $\alpha>k-1$.

The following lemma is basic for everything that follows. The need for this result led to the definition of a normal pair given above.

LEMMA 8. If $\{\varphi, \psi\}$ is a normal pair and if $m \geqq 1+\alpha$, then

$$
\int_{0}^{1}(1-\rho r)^{-m} \psi(r) d r \leqq c \frac{1}{\varphi(\rho)}(1-\rho)^{1+\alpha-m}, \quad 0 \leqq \rho<1 .
$$

Proof. With the notations of (6) and (7) we have

$$
\int_{0}^{1}(1-\rho r)^{-m} \psi(r) d r=\int_{0}^{r_{0}}+\int_{r_{0}}^{1}=I_{1}+I_{2}
$$


The first integral is bounded for $0 \leqq \rho<1$. For the second integral, if $\rho>r_{0}$ we have

$$
\begin{aligned}
I_{2}= & \int_{r_{0}}^{1}(1-\rho r)^{-m}\left(1-r^{2}\right)^{\alpha} \frac{1}{\varphi(r)} d r=\int_{r_{0}}^{\rho}+\int_{\rho}^{1} \\
\leqq & c \int_{r_{0}}^{\rho}(1-\rho r)^{-m}(1-r)^{\alpha-\varepsilon} \frac{(1-r)^{\varepsilon}}{\varphi(r)} d r \\
& +c \int_{\rho}^{1}(1-\rho r)^{-m}(1-r)^{\alpha-k} \frac{(1-r)^{k}}{\varphi(r)} d r \\
\leqq & c \frac{(1-\rho)^{\varepsilon}}{\varphi(\rho)} \int_{r_{0}}^{\rho}(1-\rho r)^{-m}(1-r)^{\alpha-\varepsilon} d r \\
& +c \frac{(1-\rho)^{k}}{\varphi(\rho)} \int_{\rho}^{1}(1-\rho r)^{-m}(1-r)^{\alpha-k} d r
\end{aligned}
$$

and the result follows from Lemma 6 , since $\alpha-\varepsilon>\alpha-k>-1$, and $m>1+\alpha-\varepsilon$. The constant $c$ is not necessarily the same at each occurrence.

2. Duality. Throughout this section $\{\varphi, \psi\}$ will denote a normal pair of weight functions (see (6), (7) in the previous section). Choose $k, \varepsilon, \alpha$ satisfying (6) and (7). We shall use the following pairing between $A_{\infty}(\varphi)$ and $A^{1}(\psi)$.

$$
(f, g)=\int f(z) g(\bar{z}) \varphi(z) \psi(z) d \sigma(z), \quad f \in A_{\infty}(\varphi), g \in A^{1}(\psi) .
$$

Note that $(f, g)$ is unchanged if $f(z) g(\bar{z})$ is replaced by $f(\bar{z}) g(z)$.

Lemma 9. Let $f=\sum a_{n} z^{n}$ and $g=\sum b_{n} z^{n}$ be polynomials. Then

$$
(f, g)=\sum a_{n} b_{n} n ! /(\alpha+1)(\alpha+2) \cdots(\alpha+n+1) .
$$

Proof.

$$
\begin{aligned}
(f, g) & =2 \int_{0}^{1} \frac{1}{2 \pi} \int_{0}^{2 \pi}\left(\sum a_{n} r^{n} e^{i n \theta}\right)\left(\sum b_{m} r^{m} e^{-i m \theta}\right)\left(1-r^{2}\right)^{\alpha} r d \theta d r \\
& =\sum a_{n} b_{n} 2 \int_{0}^{1} r^{2 n}\left(1-r^{2}\right)^{\alpha} r d r=\sum a_{n} b_{n} \int_{0}^{1} r^{n}(1-r)^{\alpha} d r
\end{aligned}
$$

from which the result follows by Lemma 4 .

REMARK. For $f=\sum a_{n} z^{n} \in A_{\infty}(\varphi), g=\sum b_{n} z^{n} \in A^{1}(\psi)$, we have

(i) $\left(f_{\rho}, g\right)=\left(f, g_{\rho}\right)=\sum a_{n} b_{n} \rho^{n} n ! /(\alpha+1) \cdots(\alpha+n+1)$,

(ii) $(f, g)=\lim _{\rho \rightarrow 1}\left(f_{\rho}, g\right)$.

The proof is similar to the above, using Lemma 3.

LEMMA 10. Let

$$
K_{w}(z)=(1+\alpha)(1-w z)^{-(2+\alpha)}, \quad z, w \in D .
$$

Then

(i) $K_{w}$ is in both $A_{0}(\varphi)$ and $A^{1}(\psi)$;

(ii) $g(w)=\left(K_{w}, g\right)$, for all $g \in A^{1}(\psi)$;

(iii) $f(w)=\left(f, K_{w}\right)$, for all $f \in A_{\infty}(\varphi)$. 
Note. The function $K_{w}$ is called the kernel function (or reproducing kernel) for the point $w$.

Proof. (i) $K_{w}$ is analytic for $|z|<|w|^{-1}$ and so is in both $A_{0}$ and $A^{1}$.

(ii) The coefficient of $z^{n}$ in the Taylor expansion of $K_{w}$ is

$$
((\alpha+1) \cdots(\alpha+n+1) / n !) w^{n}=c_{n}(\alpha) w^{n}
$$

and the result follows for polynomials from Lemma 9. The general case follows from Lemmas 1 and 3 (if two bounded linear functionals agree on a dense set then they agree identically).

(iii) For fixed $w$ the linear functional $\lambda(f)=\left(f, K_{w}\right)$ is $w^{*}$ continuous on $A_{\infty}$. As in (ii), $\lambda(f)=f(w)$ when $f$ is a polynomial. In the proof of Lemma 3(iv) it was shown that to each $f \in A_{\infty}$ there corresponds a sequence of polynomials converging to $f$ pointwise and in the $w^{*}$ topology. The result follows.

Let $\left\langle K_{w}\right\rangle$ denote the vector subspace spanned by the functions $K_{w}, w \in D$.

LeMma 11. (i) $\left\langle K_{w}\right\rangle$ is dense in $A^{1}(\psi)$ and in $A_{0}(\varphi)$;

(ii) $\left\langle K_{w}\right\rangle$ is $w^{*}$ dense in $A_{\infty}(\varphi)$.

Proof. (i) Consider first $A^{1}(\psi)$. It is equivalent to show that $\left\langle K_{w} \psi\right\rangle$ is dense in $T A^{1}$ (see (4)). By the Hahn-Banach theorem it suffices to show that if $h \in L^{\infty}(D)$ and if

$$
\int K_{w}(z) \psi(z) h(\bar{z}) d \sigma(z)=0
$$

for all $w \in D$, then $h$ annihilates all of $T A^{1}$. From (10) and (11) we have

$$
0=\sum_{n=0}^{\infty} c_{n}(\alpha) w^{n} \int z^{n} \psi(z) h(\bar{z}) d \sigma(z)
$$

for all $w \in D$. Hence $\int z^{n} \psi(z) h(\bar{z}) d \sigma(z)=0, n \geqq 0$. Thus $h$ annihilates polynomials and the result follows from Lemma 3(iii).

The proof for $A_{0}$ is similar, using the duality between $C_{0}(D)$ and $M(D)$.

(ii) The proof for $A_{\infty}(\varphi)$ is similar, using the $L^{1}(D)-L^{\infty}(D)$ duality and Lemma 3(iv).

We now come to one of our main results.

THEOREM 1. (i) The transformation $P$ defined by

$$
(P h)(w)=\int K_{w}(\bar{z}) h(z) \psi(z) d \sigma(z), \quad h \in L^{\infty}(D), w \in D,
$$

is a bounded operator mapping $L^{\infty}(D)$ onto $A_{\infty}(\varphi)$; the operator $T_{\infty} P$ is a bounded projection of $L^{\infty}(D)$ onto the subspace $T A_{\infty}$.

(ii) The transformation $P_{0}=P \mid C_{0}(D)$ is a bounded operator mapping $C_{0}(D)$ onto $A_{0}(\varphi)$; the operator $T_{0} P_{0}$ is a bounded projection of $C_{0}(D)$ onto the subspace $T A_{0}$. 
(iii) The transformation $Q$ defined by

$$
(Q \mu)(w)=\int K_{w}(\bar{z}) \varphi(z) d \mu(z), \quad \mu \in M(D), w \in D,
$$

is a bounded operator mapping $M(D)$ onto $A^{1}(\psi)$; the operator $M Q$ is a bounded projection of $M(D)$ onto the subspace $M A^{1}$.

(iv) The transformation $Q_{1}=Q \mid L^{1}(D)$ is a bounded operator mapping $L^{1}(D)$ onto $A^{1}(\psi)$; the operator $T_{1} Q_{1}$ is a bounded projection of $L^{1}(D)$ onto the subspace $T A^{1}$.

Notes. See (4) and (5) for the definitions of the operators $T_{0}, T_{1}, T_{\infty}$, and $M$. In (ii) we regard $C_{0}(D)$ as a subspace of $L^{\infty}(D)$, and in (iv) we regard $L^{1}(D)$ as a subspace of $M(D)$.

Proof. (i) From Lemmas 5 and 8 we have

$$
\begin{aligned}
|(P h)(w)| & \leqq \frac{1+\alpha}{\pi}\|h\|_{\infty} \int_{0}^{1} \int_{0}^{2 \pi}\left|1-w r e^{-i \theta}\right|^{-(2+\alpha)} \psi(r) r d \theta d r \\
& \leqq c\|h\|_{\infty} \int_{0}^{1}(1-\rho r)^{-(1+\alpha)} \psi(r) d r \leqq c\|h\|_{\infty} \frac{1}{\varphi(\rho)}
\end{aligned}
$$

where $\rho=|w|$ and the constant $c$ is not necessarily the same at each occurrence. Thus $P$ is a bounded operator mapping $L^{\infty}(D)$ into $A_{\infty}(\varphi)$. Let $f \in A_{\infty}(\varphi)$ be given. Then from Lemma 10

$$
(P(\varphi f))(w)=\left(f, K_{w}\right)=f(w) .
$$

Thus $P T_{\infty}=I$, the identity on $A_{\infty}$, and so $P$ is onto and $T_{\infty} P$ is a projection.

(ii) This will follow from (i) if it can be shown that $h \in C_{0}(D)$ implies $P_{0} h \in A_{0}(\varphi)$. Given $\varepsilon>0$ choose $R, 0<R<1$, such that $|h|<\varepsilon$ for $|z|>R$. Then

$$
|(P h)(w)| \leqq\left(\int_{|z| \leqq R}+\int_{|z|>R}\right)\left|K_{w}(\bar{z}) h(z)\right| \psi(z) d \sigma(z)=I_{1}+I_{2} .
$$

From (14), $I_{2} \leqq \varepsilon c / \varphi(w)$. Also, $I_{1} \leqq a_{R}\|h\|_{\infty}$, where $a_{R}$ is a constant depending on $R$. Hence

$$
|(P h)(w)| \varphi(w) \leqq a_{R}\|h\|_{\infty} \varphi(w)+\varepsilon c, \quad w \in D
$$

Thus

$$
\limsup _{|w| \rightarrow 1}|(P h)(w)| \varphi(w) \leqq \varepsilon c
$$

and the result follows.

(iii) For $\mu \in M(D)$,

$$
\int|(Q \mu)(w)| \psi(w) d \sigma(w) \leqq \iint\left|K_{w}(\bar{z})\right| \psi(w) d \sigma(w) \varphi(z) d|\mu|(z) .
$$

Using Leinmas 5 and 8 as above we have $\int\left|K_{w}(\bar{z})\right| \psi(w) d \sigma(w) \leqq c / \varphi(z)$. Thus $Q$ is a bounded operator from $M(D)$ into $A^{1}(\psi)$. Now let $g \in A^{1}(\psi)$. From Lemma 10 
we have $(Q(\psi g))(w)=\left(K_{w}, g\right)=g(w)$. Thus, $Q T_{1}=I$, the identity on $A^{1}(\psi)$, and so $Q$ is onto and $T_{1} Q$ is a projection.

(iv) This follows from (iii).

For the following corollary we shall use the notation

$$
\left(h_{1}, h_{2}\right)=\int h_{1}(\bar{z}) h_{2}(z) d \sigma(z), \quad h_{1} \in L^{\infty}(D), h_{2} \in L^{1}(D)
$$

COROLlaRY. We have the following direct sum decompositions:

(i) $L^{1}(D)=T A^{1} \oplus\left(T A_{\infty}\right)^{\perp}$,

(ii) $L^{\infty}(D)=T A_{\infty} \oplus\left(T A^{1}\right)^{\perp}$,

(iii) $C_{0}(D)=T A_{0} \oplus\left(M A^{1}\right)^{\perp}$,

(iv) $M(D)=M A^{1} \oplus\left(T A_{0}\right)^{\perp}$,

where

$$
\begin{aligned}
\left(T A_{\infty}\right)^{\perp} & =\left\{g \in L^{1}(D):(\varphi f, g)=0, \text { all } f \in A_{\infty}\right\}, \\
\left(T A^{1}\right)^{\perp} & =\left\{f \in L^{\infty}(D):(f, \psi g)=0, \text { all } g \in A^{1}\right\} \\
\left(M A^{1}\right)^{\perp} & =\left\{f \in C_{0}(D):(f, \psi g)=0, \text { all } g \in A^{1}\right\}, \\
\left(T A_{0}\right)^{\perp} & =\left\{\mu \in M(D): \int \varphi(z) f(\bar{z}) d \mu=0, \text { all } f \in A_{0}\right\} .
\end{aligned}
$$

Proof. (i) From Theorem 1(iv) we know that the operator $T_{1} Q_{1}$ is a bounded projection of $L^{1}(D)$ onto $T A^{1}$. It only remains to show that the null space of this projection is $\left(T A_{\infty}\right)^{\perp}$. From the definition of $T_{1} Q_{1}$ we see that $g \in L^{1}(D)$ is in the null space of $T_{1} Q_{1}$ if and only if $\int \varphi(z) K_{w}(\bar{z}) g(z) d \sigma(z)=0, w \in D$. By Lemma 11 the finite linear combinations of the kernel functions $K_{w}$ are $w^{*}$ dense in $A_{\infty}(\varphi)$. The result follows.

The proofs for (ii), (iii), and (iv) are similar.

Note that if $\psi=1$ then $A^{1}(\psi)$ becomes the space $A^{1}$ of integrable analytic functions in $D$. Thus this space is a direct summand of $L^{1}(D)$. There are many bounded projections of $L^{1}(D)$ onto $A^{1}$. For example from Theorem 1, with $\varphi(r)=\left(1-r^{2}\right)^{\alpha}$ $(\alpha>0)$ and $\psi=1$, we see that each operator

$$
\left(P_{\alpha} g\right)(w)=(1+\alpha) \int(1-w \bar{z})^{-(2+\alpha)}\left(1-|z|^{2}\right)^{\alpha} g(z) d \sigma(z), \quad 0<\alpha<\infty,
$$

is a bounded projection from $L^{1}(D)$ onto $A^{1}$.

Lindenstrauss and Pełczyński [6] use the existence of bounded projections from $L^{1}(D)$ onto $A^{1}$ to show that $A^{1}$ is isomorphic to the Banach space $l^{1}$. It would be interesting to find explicitly the functions in $A^{1}$ that correspond to the natural basis in $l^{1}$.

LEMMA 12. If we use the pairing between $L^{1}(D)$ and $L^{\infty}(D)$ given by (15), then the operator $T_{\infty} P: L^{\infty}(D) \rightarrow L^{\infty}(D)$ is the adjoint of $T_{1} Q_{1}: L^{1}(D) \rightarrow L^{1}(D)$. 
If we define the pairing between $C_{0}(D)$ and $M(D)$ by

$$
(f, \mu)=\int f(\bar{z}) d \mu(z), \quad f \in C_{0}(D), \mu \in M(D),
$$

then the operator $M Q: M(D) \rightarrow M(D)$ is adjoint to $T_{0} P_{0}: C_{0}(D) \rightarrow C_{0}(D)$.

The proof is a straightforward calculation which we omit.

Corollary. The subspace $M A^{1}$ is $w^{*}$ closed in $M(D)$.

Proof. The operator $M Q$ is $w^{*}$ continuous since it is an adjoint operator. Also, $M A^{1}$ is the null space of $I-M Q$, hence is $w^{*}$ closed.

Note. The proof could also be given directly, using the fact that a subspace of the conjugate space of a separable Banach space is $w^{*}$ closed if and only if it is sequentially closed.

We come now to our second main result. The symbol $\cong$ denotes topological isomorphism.

THEOREM 2. Using the pairing given by (8) we have

(i) $A_{0}(\varphi)^{*} \cong A^{1}(\psi)$,

(ii) $A^{1}(\psi)^{*} \cong A_{\infty}(\varphi)$.

More precisely, if $g \in A^{1}(\psi)$ and if we define $\lambda_{g}(f)=(f, g), f \in A_{0}(\varphi)$, then $\lambda_{g} \in A_{0}(\varphi)^{*}$ and $\left\|\lambda_{g}\right\| \leqq\|g\|_{\psi}$. Conversely, given $\lambda \in A_{0}(\varphi)^{*}$ then there is a unique $g \in A^{1}(\psi)$ such that $\lambda=\lambda_{g}$. Also, $\|g\|_{\psi} \leqq\|Q\|\|\lambda\|$.

Furthermore, if $f \in A_{\infty}(\varphi)$ and if we define $\lambda_{f}(g)=(f, g), g \in A^{1}(\psi)$, then $\lambda_{f} \in A^{1}(\psi)^{*}$ and $\left\|\lambda_{f}\right\| \leqq\|f\|_{\varphi}$. Conversely, given $\lambda \in A^{1}(\psi)^{*}$ then there is a unique $f \in A_{\infty}(\varphi)$ such that $\lambda=\lambda_{f}$. Also, $\|f\|_{\varphi} \leqq\|P\|\|\lambda\|$.

Proof. (i) It is trivial that if $g \in A^{1}$ then $\lambda_{g} \in A_{0}^{*}$. We also have uniqueness: if $\lambda_{g}(f)=0$ for all $f \in A_{0}$, then $g=0$. Indeed, from Lemma $10, g(w)=\lambda_{g}\left(K_{w}\right)$.

Now let $\lambda \in A_{0}^{*}$ be given. Since $T_{0}$ is an isometric embedding of $A_{0}$ into $C_{0}(D)$, there exists $\mu \in M(D)$ with $\|\mu\|=\|\lambda\|$ and $\lambda(f)=(\varphi f, \mu)$ for all $f \in A_{0}$ (see (16)). Let $g(w)=\lambda\left(K_{w}\right)$. Then

$$
g(w)=\lambda\left(K_{w}\right)=\int K_{w}(\bar{z}) \varphi(z) d \mu(z)=(Q \mu)(w) .
$$

By Theorem 1(iii), $g \in A^{1}$ and $\|g\|_{\psi} \leqq\|Q\|\|\mu\|=\|Q\|\|\lambda\|$. From Lemma 10 we see that $\lambda_{g}\left(K_{w}\right)=g(w)$ for $w \in D$. Hence $\lambda=\lambda_{g}$ on the subspace spanned by the kernel functions $K_{w}$, and this is all of $A_{0}$ by Lemma 11 .

(ii) The proof is similar and will be omitted.

Note. For the special case when $\varphi(r)=\left(1-r^{2}\right)^{b}, b>0$, the duality was given in a different form by Duren, Romberg, and Shields [2, Theorems 7 and 11]. Their proof was based on fractional integration.

It follows from Theorem 2 that $A_{\infty}(\varphi)$ is isomorphic to the second dual of $A_{0}(\varphi)$. Actually Rubel and Shields [7] have shown that $A_{\infty}$ is always isometrically isomorphic to the second dual of $A_{0}$, even when $\varphi$ is not a normal weight function. 
Their result was generalized by Shapiro, Shields, and Taylor [8]. In [7] the intermediate space is only exhibited as a quotient space of $L^{1}(D)$, rather than as a subspace which is a direct summand. It seems doubtful that bounded projections always exist when $\varphi$ is not normal (see $\S 4$ ).

3. Multipliers. Throughout this section $\{\varphi, \psi\}$ will denote a normal pair of weight functions as defined in $\S 1,(6)$ and (7).

Let $A$ be a sequence space. A sequence $\left\{\lambda_{n}\right\}$ is said to be a multiplier on $A$ if $\left\{\lambda_{n} a_{n}\right\} \in A$ whenever $\left\{a_{n}\right\} \in A$. The spaces $A_{0}(\varphi), A_{\infty}(\varphi), A^{1}(\psi)$ may be regarded as sequence spaces (Taylor coefficients). In each of these three spaces evaluation of the $n$th Taylor coefficient is a bounded linear functional. A little calculation shows that any (Taylor coefficient) multiplier on one of these spaces has a closed graph and hence is a bounded linear transformation.

We require three lemmas.

LEMMA 13. There is a constant $c$ such that $\varphi\left(r^{2}\right) \leqq c \varphi(r), 0 \leqq r<1$.

Proof. With the notations of (6) we have

$$
\frac{\varphi\left(r^{2}\right)}{\varphi(r)}=(1+r)^{k} \frac{\varphi\left(r^{2}\right)}{\left(1-r^{2}\right)^{k}} \frac{(1-r)^{k}}{\varphi(r)} \leqq(1+r)^{k}<2^{k}, \quad r_{0} \leqq r^{2}<1,
$$

since $\varphi(r) /(1-r)^{k}$ is increasing for $r_{0} \leqq r<1$. Clearly $\varphi\left(r^{2}\right) / \varphi(r)$ is bounded for $r^{2} \leqq r_{0}$ and the result follows.

LEMMA 14. We have $f \in A_{\infty}(\varphi)$ if and only if

$$
\max _{|z|=r} \varphi(z)\left|f^{\prime}(z)\right| \leqq \frac{c}{1-r}, \quad 0 \leqq r<1 .
$$

Proof. If $f \in A_{\infty}$ then

$$
f^{\prime}(w)=\frac{1}{2 \pi i} \int_{|\zeta|=\rho} \frac{f(\zeta)}{(\zeta-w)^{2}} d \zeta, \quad|w|<\rho<1 .
$$

Thus,

$$
\left|f^{\prime}(w)\right| \leqq M_{\infty}(f, \rho) \frac{1}{2 \pi} \int_{0}^{2 \pi} \frac{\rho d t}{\left|\rho e^{i t}-w\right|^{2}} \leqq\|f\|_{\varphi} \frac{1}{\varphi(\rho)} \frac{1}{\rho^{2}-|w|^{2}} .
$$

It suffices to consider $w$ bounded away from zero, say $\frac{1}{4} \leqq|w|<1$. The result follows from Lemma 13 if we take $\rho=|w|^{1 / 2}$.

Conversely, assume that (17) holds. We use the notations of (6). Choose $w$ so that $r_{0}<|w|<1$. Let $w_{0}=r_{0} w /|w|$, and let $r=|w|$. Then

$$
\begin{aligned}
\left|f(w)-f\left(w_{0}\right)\right| & =\left|\int_{w_{0}}^{w} f^{\prime}(\zeta) d \zeta\right| \leqq c \int_{r_{0}}^{r} \frac{1}{1-t} \frac{1}{\varphi(t)} d t \\
& =c \int_{r_{0}}^{r} \frac{1}{(1-t)^{1+\varepsilon}} \frac{(1-t)^{\varepsilon}}{\varphi(t)} d t \\
& \leqq c \frac{(1-r)^{\varepsilon}}{\varphi(r)} \int_{0}^{r} \frac{d t}{(1-t)^{1+\varepsilon}} \leqq \frac{c}{\varepsilon \varphi(r)},
\end{aligned}
$$

and the result follows. 
REMARK. The proof of the converse really shows that the operator of integration is a bounded linear transformation from the space of functions satisfying (17) onto the subspace of $A_{\infty}$ consisting of functions that vanish at the origin.

Let $\left\{\lambda_{n}\right\}, n=0,1, \ldots$, be given and let $(s)$ denote the space of all sequences of complex numbers. We define three operators

$$
S_{0}: A_{0}(\varphi) \rightarrow(s), \quad S_{1}: A^{1}(\psi) \rightarrow(s), \quad S_{\infty}: A_{\infty}(\varphi) \rightarrow(s),
$$

by the single formula $T\left(\sum a_{n} z^{n}\right)=\left\{\lambda_{n} a_{n}\right\}$. We shall say that $S_{0}$ is bounded (more correctly, is a bounded operator on $A_{0}$ ) if $S_{0} A_{0} \subset A_{0}$ (as noted earlier it then follows from the closed graph theorem that $S_{0}$ is a bounded linear transformation from $A_{0}$ into itself). We make similar definitions for $S_{1}$ and $S_{\infty}$.

LEMMA 15. If any one of the three operators $S_{0}, S_{1}, S_{\infty}$ is bounded then all are bounded. In this case $S_{0}^{*}=S_{1}$ and $S_{1}^{*}=S_{\infty}$.

Proof. Suppose first that $S_{0}$ is bounded. Let $f=\sum a_{n} z^{n} \in A_{0}$ and let $g=\sum b_{n} z^{n}$ be a polynomial. Then from Lemma 9 and the remark following it we have

$$
\left(S_{0} f, g\right)=\sum\left(a_{n} \lambda_{n}\right) b_{n} c_{n}(\alpha)=\sum a_{n}\left(\lambda_{n} b_{n}\right) c_{n}(\alpha)=\left(f, S_{1} g\right)
$$

Thus $S_{1}=S_{0}^{*}$ on the polynomials. Thus $S_{1}$ coincides with a bounded operator on a dense subset and so $S_{1}$ is bounded and $S_{1}=S_{0}^{*}$.

Similarly, one shows that if $S_{1}$ is bounded then $S_{\infty}=S_{1}^{*}$.

Finally, suppose that $S_{\infty}$ is bounded. We wish to show that $S_{0}$ is bounded. Since $S_{0}$ is the restriction of $S_{\infty}$ to $A_{0}$, it will be sufficient to prove that $S_{\infty} A_{0} \subset A_{0}$. But this is trivial since $S_{\infty}$ maps polynomials into polynomials and these are dense in $A_{0}$ (Lemma 3).

THEOREM 3. The sequence $\Lambda=\left\{\lambda_{n}\right\}, n=0,1, \ldots$, is a multiplier on one of the spaces $A_{0}(\varphi), A^{1}(\psi), A_{\infty}(\varphi)$ if and only if it is a multiplier on all of them. This happens if and only if

(i) the power series $h(z)=\sum \lambda_{n} z^{n}$ converges for $|z|<1$, and

(ii) $M_{1}\left(h^{\prime}, r\right)=O(1 /(1-r))$.

Proof. We first show that if (i) and (ii) hold then $\Lambda$ is a multiplier on $A_{\infty}$. Next we shall show that if $\Lambda$ is a multiplier on $A^{1}$ then (i) and (ii) hold. In view of Lemma 15 this will establish the theorem.

First assume that conditions (i) and (ii) are satisfied. It will be convenient to rewrite $\Lambda$ as an integral operator. For $f(z)=\sum a_{n} z^{n}$ in $A_{\infty}$ we have

$$
\sum \lambda_{n} a_{n} \zeta^{n} w^{n}=(\Lambda f)(\zeta w)=\frac{1}{2 \pi} \int_{0}^{2 \pi} h\left(w e^{i t}\right) f\left(\zeta e^{-i t}\right) d t .
$$

Letting $g=\Lambda f$ we have

$$
\zeta g^{\prime}(\zeta w)=\frac{1}{2 \pi} \int_{0}^{2 \pi} h^{\prime}\left(w e^{i t}\right) f\left(\zeta e^{-i t}\right) e^{i t} d t
$$


and so

$$
\left|\zeta g^{\prime}(\zeta w)\right| \leqq\|f\|_{\varphi} \frac{1}{\varphi(\zeta)} M_{1}\left(h^{\prime},|w|\right) \leqq c\|f\|_{\varphi} \frac{1}{\varphi(\zeta)} \frac{1}{1-|w|}
$$

Taking $\zeta=w$ and applying Lemmas 13 and 14 we see that $g \in A_{\infty}$.

Conversely, assume that $\Lambda$ is a multiplier on $A^{1}(\psi)$. The function $\sum z^{n} / n^{2}$ is in $A^{1}$, hence so is $\sum \lambda_{n} z^{n} / n^{2}$. In particular this series converges for $|z|<1$, from which (i) follows.

Next note that $\Lambda$ is a bounded linear transformation on $A^{1}$ (see the remarks preceding Lemma 13). We use the notations of (6) and (7). Let $m \geqq \alpha+1$ be a positive integer, let $G=z^{m} h$, and let $f=m ! /(1-z)^{m+1}$. Then a little calculation shows that $\Lambda f=G^{(m)}$, and therefore

$$
\left(\Lambda f_{\rho}\right)(z)=(\Lambda f)(\rho z)=G^{(m)}(\rho z), \quad 0<\rho,|z|<1 .
$$

Hence

$$
\left\|\left(G^{(m)}\right)_{\rho}\right\|_{\psi} \leqq\|\Lambda\|\left\|f_{\rho}\right\|_{\psi}
$$

Now if $z=r e^{i \theta}$ we have

$$
\begin{aligned}
\left\|f_{\rho}\right\|_{\psi} & =\frac{m !}{\pi} \iint \frac{d \theta}{|1-\rho z|^{m+1}} \psi(r) r d r \\
& \leqq c \int \frac{1}{(1-r \rho)^{m}} \psi(r) d r \leqq c \frac{1}{\varphi(\rho)}(1-\rho)^{1+\alpha-m}
\end{aligned}
$$

by Lemmas 5 and 8 . Hence from (18) we have

$$
\begin{aligned}
c \frac{1}{\varphi(\rho)}(1-\rho)^{1+\alpha-m} & \geqq\left\|\left(G^{(m)}\right)_{\rho}\right\|_{\psi}=2 \int_{0}^{1} M_{1}\left(G^{(m)}, \rho r\right) \psi(r) r d r \\
& \geqq 2 \int_{\rho}^{1} M_{1}\left(G^{(m)}, \rho r\right)\left(1-r^{2}\right)^{\alpha-\varepsilon} \frac{(1-r)^{\varepsilon}}{\varphi(r)} r d r \\
& \geqq M_{1}\left(G^{(m)}, \rho^{2}\right) \frac{(1-\rho)^{\varepsilon}}{\varphi(\rho)} \int_{\rho}^{1}(1-r)^{\alpha-\varepsilon} d r \\
& \geqq M_{1}\left(G^{(m)}, \rho^{2}\right) \frac{(1-\rho)^{\alpha+1}}{\varphi(\rho)} .
\end{aligned}
$$

Hence

$$
M_{1}\left(G^{(m)}, \rho\right) \leqq c /(1-\rho)^{m} .
$$

By a result of Hardy and Littlewood [4, Theorem 47, p. 435] this is equivalent to $M_{1}\left(G^{\prime}, \rho\right)=O(1 /(1-\rho))$ from which (ii) follows. This completes the proof.

REMARKS. The theorem for $A^{1}(\psi)$, when $\psi(r)=(1-r)^{\beta}, \beta>-1$, is in a paper of Duren and Shields $\left[3\right.$, Corollary to Theorem 3, p. 75]. The spaces $A^{1}\left((1-r)^{\beta}\right)$ are all isomorphic to one another under a modified form of fractional integration. For example, if $\lambda_{n}=1 /(n+1)$, then $S_{1}$ maps $A^{1}(1)$ isomorphically onto $A^{1}(1-r)$. See [2, Theorem 5] for a complete statement. However we do not know whether all 
the spaces $A^{1}(\psi)$ are isomorphic to $A^{1}(1)$, when $\psi$ is part of a normal pair of weight functions.

Zygmund [9] has found the Fourier series multipliers of the space $\Lambda_{\alpha}$ of continuous periodic functions satisfying a Lipschitz condition of order $\alpha$, and of the subspace $\lambda_{\alpha}$ of functions for which $|x-y|^{\alpha}|f(x)-f(y)| \rightarrow 0$ as $|x-y| \rightarrow 0$. It is known that $f$ is in $\Lambda_{\alpha}$ (or $\lambda_{\alpha}$ ) if and only if

$$
\sum_{-\infty}^{\infty} n \hat{f}(n) r^{|n|} e^{i n \theta}=O\left((1-r)^{\alpha-1}\right) \quad\left(\text { or } o\left((1-r)^{\alpha-1}\right)\right)
$$

where $\hat{f}$ denotes the Fourier coefficients of $f$ (see [10, Chapter VII, Theorem 5.1]). It is also known that if $f$ is in $\Lambda_{\alpha}$ (or $\lambda_{\alpha}$ ), then so is the projection $\sum_{0}^{\infty} \hat{f}(n) e^{i n \theta}$ (see [1, Chapter 5, Theorem 5.8]). Thus our multiplier result for the spaces $A_{0}(\varphi)$ and $A_{\infty}(\varphi)$, for the special case $\varphi(r)=(1-r)^{\beta}, 0<\beta<1$, could be deduced from Zygmund's results. This suggests studying the relation between our more general growth classes and moduli of continuity more general than Lipschitz conditions, and extending Zygmund's result (and the projection theorem) to these more general classes of continuous functions.

The spaces $A_{\infty}\left((1-r)^{\beta}\right)$ are all isomorphic to one another under a modified form of fractional integration (see Hardy and Littlewood [4, Theorem 39, p. 425]). However, we do not know whether all the spaces $A_{\infty}(\varphi), \varphi$ normal, are isomorphic to one another. Similar remarks apply to $A_{0}$.

The space of multipliers, that is, the space of analytic functions $h(z)$ satisfying conditions (i) and (ii) of Theorem 3, forms a commutative Banach algebra with identity under coordinatewise multiplication of the Taylor coefficients (Hadamard multiplication) and with the operator norm. It might be interesting to study this algebra in more detail.

4. Concluding remarks. The outstanding problem left unsolved in this discussion is the extent to which the results remain true when $\varphi$ is not a normal weight function. Thus we do not know whether Theorem 1, on the existence of bounded projections, and Theorem 3, characterizing the Taylor coefficient multipliers, remain valid when $\varphi$ tends to zero too slowly or too rapidly, for example when

$$
\varphi(r)=r / \log (1-r)^{-1},
$$

or

$$
\varphi(r)=\exp \left(-(1-r)^{-1}\right) .
$$

There is some reason to think that the results may be false in these cases. Our proofs depended on certain elementary calculations, culminating in Lemma 8 . The assertion of this lemma is false when $\varphi$ is given by (19) and $\psi(r)=1 / \varphi(r)$. Furthermore, in this case the operator $P_{0}$ defined in Theorem 1(ii) is not a bounded operator from $C_{0}(D)$ to $A_{0}(\varphi)$, and the space $A^{1}(\psi)$ is not isomorphic, in the pairing (8), to $A_{0}(\varphi)^{*}$, as the next two results show. 
THEOREM 4. If $\varphi(r)$ is given by (19) and if $\psi(r)=1 / \varphi(r)$, then each $g \in A^{1}(\psi)$ induces a bounded linear functional $\lambda_{g}$ on $A_{0}(\varphi)$ by the formula

$$
\lambda_{g}(f)=(f, g)=\int f(z) g(\bar{z}) d \sigma(z),
$$

and $\left\|\lambda_{g}\right\| \leqq\|g\|_{\psi}$. However, there are additional linear functionals on $A_{0}(\varphi)$ that are not of this form.

Proof. Assume that all continuous linear functionals on $A_{0}$ are of the form (21). Then the two norms are equivalent on $A^{1}$ and so there is a constant $c$ such that

$$
\|g\|_{\psi} \leqq c\left\|\lambda_{g}\right\|, \quad g \in A^{1}(\psi) .
$$

Here $K_{w}(z)=(1-w z)^{-2}$. Let $\lambda_{w}$ denote $\lambda_{g}$ when $g=K_{w}$. Then from (21) and Lemma $10, \lambda_{w}(f)=f(w), f \in A_{0}(\varphi), w \in D$. Hence, if $\rho=|w|$,

$$
\left\|\lambda_{w}\right\|=\sup _{\|f\| \varphi \leqq 1}|f(w)| \leqq \frac{1}{\varphi(w)}=\frac{1}{\rho} \log \frac{1}{1-\rho} .
$$

On the other hand, if $z=r e^{i \theta}$,

$$
\begin{aligned}
\left\|K_{w}\right\|_{\psi} & =\frac{1}{\pi} \iint \frac{1}{|1-w z|^{2}} \psi(r) r d r d \theta \\
& =2 \int_{0}^{1} \frac{1}{1-\rho^{2} r^{2}} \log \frac{1}{1-r} d r \\
& \geqq \int_{0}^{1} \frac{1}{1-\rho r} \log \frac{1}{1-\rho r} d r=\frac{1}{2 \rho}\left(\log \frac{1}{1-\rho}\right)^{2} .
\end{aligned}
$$

Finally, (23) and (24) contradict (22) as $\rho \rightarrow 1$, which completes the proof.

Note that these calculations show that the conclusion to Lemma 8 is not valid here.

COROLlary. If $\varphi(r)$ is given by (19), if $\psi(r)=1 / \varphi(r)$, and if the transformation $P_{0}$ is defined as in Theorem 2(ii), then $P_{0}$ is not a bounded linear operator from $C_{0}(D)$ to $A_{0}(\varphi)$.

Proof. If $P_{0}$ were bounded then the proof of Theorem 2 would show that $A^{1}(\psi) \cong A_{0}(\varphi)^{*}$ in the pairing (21), contradicting Theorem 4. (The proof of Theorem 2 also requires Lemmas $9,10,11$, which are still valid in this case.)

The case of weight functions $\varphi(r)$ that go rapidly to zero, as in (20), is somewhat different in that there is no integrable weight function $\psi$ such that $\varphi \psi(r)=\left(1-r^{2}\right)^{\alpha}$, $\alpha>-1$. The calculations that established Theorem 3 on multipliers fail when $\varphi$ is given by (20). We conjecture that the main results of the paper do not hold for $A_{0}(\varphi)$ and $A_{\infty}(\varphi)$ in this case.

It would be interesting to find the multipliers on $A_{0}(\varphi)$ and $A_{\infty}(\varphi)$ in cases like (19) and (20). Also, it would be interesting to know whether $A_{\infty}(\varphi)$ and $A_{0}(\varphi)$ are isomorphic to $A_{\infty}(1-r)$ and $A_{0}(1-r)$, respectively, in these cases.

Some recent results of Joel $\mathrm{H}$. Shapiro are relevant here. First he notes that if $\{\varphi, \psi\}$ is a normal pair, then there are real numbers $b>a>-1$ such that

$$
c_{1}(1-r)^{b} \leqq \psi(r) \leqq c_{2}(1-r)^{a}, \quad 0 \leqq r<1 .
$$


(A more precise statement can be made, analogous to Lemma 7.) Then he shows that if $\psi(r)$ satisfies

$$
\psi(r) \geqq c(1-r)^{-1}\left[\log (1-r)^{-1}\right]^{-2}, \quad r_{0}<r<1,
$$

there is no bounded projection of $L^{1}(D)$ onto $T_{1} A^{1}(\psi)$.

The space $H^{\infty}$ of bounded analytic functions in the unit disc, i.e., the space $A_{\infty}(\varphi)$ for $\varphi \equiv 1$, is not included in our work. In this case there is no associated space $A_{0}$, and Rickart has asked whether $H^{\infty}$ is isomorphic to the second conjugate of some Banach space. L. Zalcman has pointed out to us that there are no bounded projections of $L^{\infty}(D)$ onto $H^{\infty}$. The argument goes like this. Let $h^{\infty}$ denote the space of bounded (complex-valued) harmonic functions in the unit disc. Then $h^{\infty}$ is a closed subspace of $L^{\infty}(D)$, and $H^{\infty} \subset h^{\infty}$. If there were a bounded projection from $L^{\infty}(D)$ onto $H^{\infty}$, then the restriction to $h^{\infty}$ would yield a bounded projection from $h^{\infty}$ onto $H^{\infty}$. But $h^{\infty}$ is isometric to $L^{\infty}(|z|=1)$ and it is a known fact that there are no bounded projections from $L^{\infty}(|z|=1)$ onto $H^{\infty}$ (see the last theorem in Chapter 9 of K. Hoffman's book Banach spaces of analytic functions).

Finally, we note that, although we have worked in the framework of the $L^{1}, L^{\infty}$ duality, we could have considered $L^{p}$. For instance, if $\varphi(r)=\left(1-r^{2}\right)^{\beta}$ then we could take $\psi=\varphi$. In this case the projection operators $T_{\infty} P$ and $T_{1} Q_{1}$ described in Theorem 2(i), (iv) coincide on polynomials and so, by the Riesz-Thorin interpolation theorem, they define a bounded projection on each space $L^{p}, 1 \leqq p \leqq \infty$.

\section{REFERENCES}

1. P. L. Duren, Theory of $H^{p}$ spaces, Academic Press, New York, 1970.

2. P. L. Duren, B. W. Romberg and A. L. Shields, Linear functionals on $H^{p}$ spaces with $0<p<1$, J. Reine Angew. Math. 238 (1969), 32-60.

3. P. L. Duren and A. L. Shields, Coefficient multipliers of $H^{p}$ and $B^{p}$ spaces, Pacific J. Math. 32 (1970), 69-78. MR 41 \#485.

4. G. H. Hardy and J. E. Littlewood, Some properties of fractional integrals. II, Math. Z. 34 (1932), 403-439.

5. E. Landau, Darstellung und Begründung einiger neuerer Ergebnisse der Funktionentheorie, Springer-Verlag, Berlin, 1929.

6. J. Lindenstrauss and A. Pełczyński, Contributions to the theory of the classical Banach spaces (preprint).

7. L. A. Rubel and A. L. Shields, The second duals of certain spaces of analytic functions, J. Austral. Math. Soc. 11 (1970), 276-280.

8. J. H. Shapiro, A. L. Shields and G. D. Taylor, The second duals of some function spaces (preprint).

9. A. Zygmund, On the preservation of classes of functions, J. Math. Mech. 8 (1959), 889-895; erratum, ibid. 9 (1960), 663. MR 22 \#8277.

10. - Trigonometric series, Vols. 1, 2, 2nd ed., Cambridge Univ. Press, London, 1968. MR 38 \#4882.

Department of Mathematics, University of Michigan, Ann Arbor, Michigan 48104

Department of Mathematics, Syracuse University, Syracuse, New York 13210 